Revue pluridisciplinaire d'études médiévales

\title{
Édifier une église pour construire sa renommée. L'église Saint-Hilaire des seigneurs de Semur-en-Brionnais
}

Anelise Nicolier

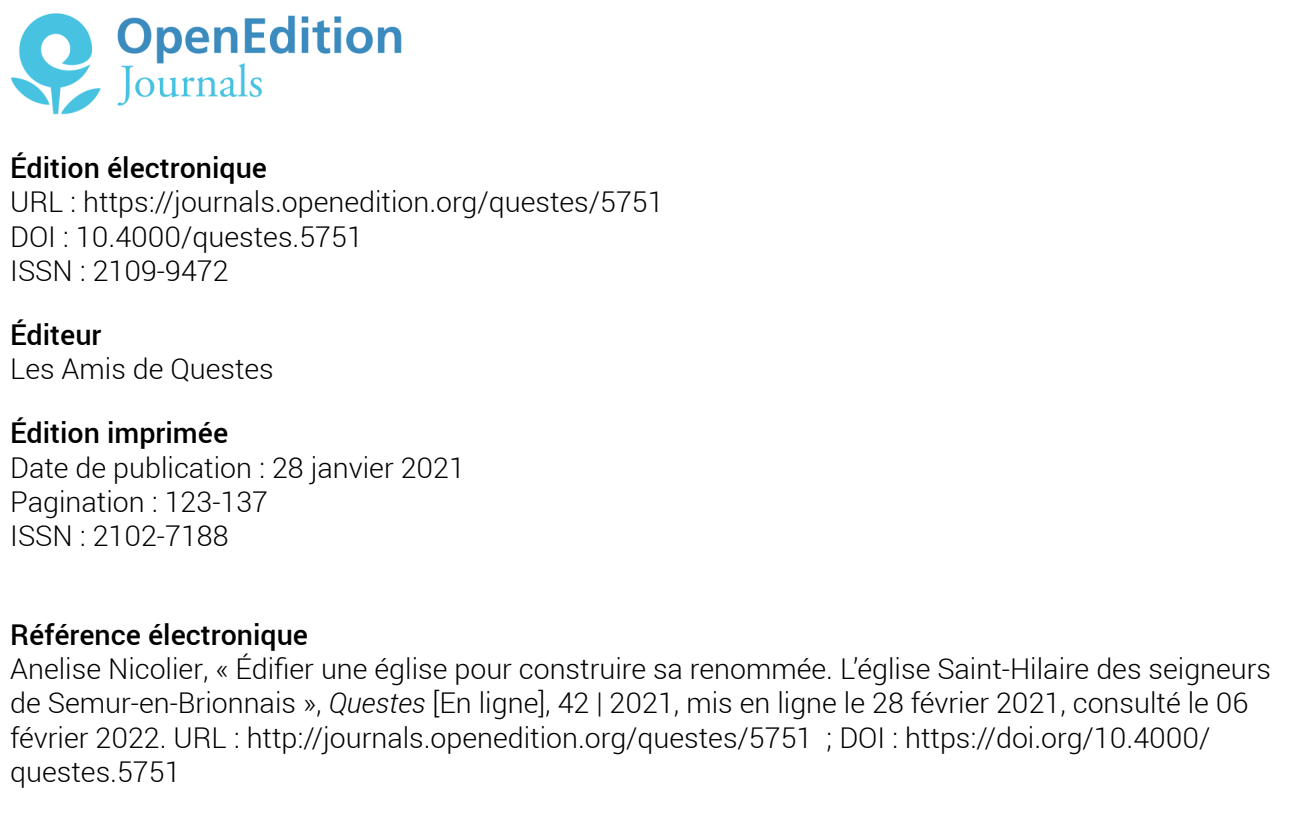

(C) Association des amis de «Questes » 


\title{
Édifier une église pour construire sa renommée. L'église Saint-Hilaire des seigneurs de Semur-en-Brionnais
}

\author{
Anelise NICOLIER
}

UMR 5138 Archéologie et Archéométrie

À la fin du $\mathrm{X}^{\mathrm{e}}$ siècle, Joceran, un fils cadet du seigneur de Chamelet, dans la vallée de l'Azergues, fonde la seigneurie de Semur, en Brionnais, au sud-ouest de l'actuelle Saône-et-Loire ${ }^{1}$. Seigneurs ambitieux, les héritiers de Joceran nouent des alliances prestigieuses et installent les cadets aux plus hautes charges ecclésiastiques. Vers 1038, par exemple, le seigneur Damas $I^{\text {er }}$ place son jeune fils Hugues, né au château en 1024, auprès des moines de Cluny. Hugues devient grand-prieur de l'abbaye dix ans plus tard et est élu abbé en février 1049. Son abbatiat de soixante ans est souvent considéré comme l'apogée de Cluny, et Hugues est proclamé saint le 6 janvier 1120 par le pape Calixte II. Si le destin d'Hugues est particulièrement remarquable, la famille compte, au cours des deux siècles de son histoire, plusieurs membres éminents qui lui permettent d'asseoir rapidement et durablement son autorité sur son territoire, bordé par le comté de Forez au sud et les terres charolaises des comtes de Chalon au nord.

La seigneurie est centrée sur un château édifié vers l'an mil et dont on conserve d'importants vestiges. Sans doute antérieure, une chapelle castrale est attestée dans les textes dès $1120^{2}$. Dans la seconde moitié du XII ${ }^{\mathrm{e}}$ siècle, la famille entreprend la reconstruction de cette chapelle, qui a acquis entre temps le statut

\footnotetext{
${ }^{1}$ Anelise Nicolier, La construction d'un paysage monumental religieux en Brionnais à l'époque romane, Thèse de Doctorat, Université Lyon 2, 2015, t. 1, vol. 1, p. 46-50.

${ }^{2}$ La chapelle Saint-Hilaire est mentionnée dans la charte 270 du Cartulaire de Marcigny-surLoire (1045-1144), éd. Jean Richard, Dijon, Bernigaud et Privat, 1957.
} 
d'église paroissiale. Cette église romane est actuellement conservée à quelques mètres seulement du château. L'étude du décor sculpté de son portail principal, puis de son architecture, montrera que la famille a utilisé l'art à des fins politiques pour accroître sa renommée.

\section{Le décor sculpté, témoin subtil de la grandeur d'un lignage}

Dans l'église de Semur-en-Brionnais, ce n'est pas l'iconographie du portail occidental qui a d'abord retenu l'attention des historiens de l'art, mais plutôt son style jugé désastreux et peu digne d'intérêt. En 1894, par exemple, Édouard Jeannez évoquant le linteau du portail affirme que « l'art n'a rien de commun avec cette frise ${ }^{3} »$. Son jugement sur le tympan est tout aussi définitif : « Rien d'inspiré ni d'artistique dans cette composition ». Quant à André Rhein, il déplore, en 1920, « une réelle monotonie et une lourdeur d'exécution, bien éloignée de l'admirable maîtrise de l'auteur du portail de Charlieu ${ }^{4} \gg$. Enfin, quel historien d'art aurait été tenté par l'étude du portail une fois que Raymond Oursel aura affirmé, en 1956, que «Ce portail n'est pas une œuvre d'art ${ }^{5}$ »? Il a fallu attendre la fin des années 2000 pour que Gil Fishhof propose enfin une analyse du programme iconographique qui, laissant de côté la question esthétique, le considère pour luimême ${ }^{6}$.

\section{L'iconographie inattendue du portail occidental}

Au tympan figure un Christ en majesté entouré du Tétramorphe, tandis qu'un Agneau portant le nimbe crucifère est sculpté à la clé du rouleau

\footnotetext{
${ }^{3}$ Édouard Jeannez, dans Félix Thiollier, L'art roman à Charlieu et dans les régions voisines, Montbrison, Société archéologique du Forez, 1894, p. 64.

${ }^{4}$ André Rhein, «Église de Semur-en-Brionnais », Bulletin monumental, 79, 1920, p. 183-196.

${ }^{5}$ Raymond Oursel, Les Églises romanes de l'Autunois et du Brionnais. Ancien grand archidiaconé d'Autun. Cluny et sa région, Mâcon, Protat, 1956, p. 294-295.

${ }^{6}$ Gil Fishhof, "The Master of the Tympanum of Saint-Hilaire in Semur-en-Brionnais : Rethinking the Meaning of Style and Concepts of Decline in Burgundian Romanesque Sculpture », Annales de Bourgogne, 84, 2012, fasc. 3, p. 245-279.
} 
d'archivolte (fig. 1). Au linteau, on s'attendrait à la représentation des apôtres comme au portail ouest de Charlieu, ou éventuellement des Vingt-Quatre Vieillards de l'Apocalypse comme sur la voussure du portail d'Anzy-le-Duc, mais le thème est, de manière exceptionnelle, l'intervention de saint Hilaire au concile de Séleucie. C'est un détour par la littérature ecclésiastique de l'époque, nommément au sujet de la querelle des ariens, qui rend compte de cette étrange intrusion dans l'iconographie céleste du portail. La première mention d'Hilaire en tant qu'évêque de Poitiers date de 356. Il prend alors part au concile de Béziers, au cours duquel il affirme ses positions anti-ariennes, ce qui lui vaut d'être exilé en Phrygie, tout en conservant son titre d'évêque. Pendant son exil, Hilaire, contrairement à d'autres évêques, jouit de sa liberté de mouvement et enrichit sa formation culturelle et doctrinale, notamment par la lecture des écrivains chrétiens de langue grecque. Il approfondit en particulier sa connaissance de l'arianisme, et c'est de ces trois ans d'exil que datent ses œuvres les plus importantes : De Trinitate et De Synodis. En 359, l'empereur Constance réunit deux conciles : l'un pour l'Occident se tient à Rimini en Italie, l'autre pour l'Orient se déroule à Séleucie d'Isaurie en Asie Mineure, et Hilaire choisit de s'y rendre. Trois sources seulement relatent l'intervention d'Hilaire lors de ce concile : le Guide du pèlerin de Saint-Jacques de Compostelle rédigé peu après 1139, le Speculum historiale de Vincent de Beauvais commandé à son auteur en 1246 par Louis IX, et la Légende dorée écrite par Jacques de Voragine vers $1255^{7}$. Par ailleurs, le chercheur Horst Fuhrmann a montré que l'affaire était connue en Occident au Moyen Âge, et qu'elle circulait notamment sous forme d' exemplum ${ }^{8}$.

\footnotetext{
${ }^{7}$ Le Guide du pèlerin de Saint-Jacques de Compostelle. Texte latin du XII ${ }^{e}$ s., édité et traduit en français d'après les manuscrits de Compostelle et de Ripoll, éd. Jeanne Vieillard, Mâcon, Protat frères, 1963, p. 60-63. Jacques de Voragine, La Légende dorée, traduit du latin par Théodore de Wyzewa, Paris, Éditions du Seuil, 1998, p. 79-81.

${ }^{8}$ Horst Fuhrmann, « Die Fabel von Papst Leo und Bischof Hilarius. Vom Ursprung und der Erscheinungsform einer historischen Legende », Archiv für Kulturgeschichte, 43/2, 1961, p. 125-162.
} 
C'est à la lumière de la Légende dorée que les scènes sculptées sur le linteau de Semur prennent tout leur sens. Le texte commence ainsi : «À cette époque, le pape Léon, perverti par l'erreur des hérétiques, convoqua un concile général des évêques, et Hilaire se joignit à eux sans avoir été invité. À cette nouvelle, le pape interdit à quiconque de se lever pour lui faire une place ». Ce sont ces lignes qu'illustre la scène de gauche sous le rang de palmettes : trois évêques assis symbolisent les participants au concile, Hilaire debout à leur droite (fig. 2). Un vif échange s'engage alors entre le pape et Hilaire, lequel exprime clairement le fait qu'il ne reconnaît pas l'autorité du pape. Mais la discussion est subitement interrompue car un besoin pressant oblige le pape à quitter précipitamment la salle du concile. Le texte dit : «Parti au lieu retiré où l'appelait la nature, le pape périt de dysenterie, et c'est en répandant là toutes ses entrailles qu'il finit misérablement sa vie ». La mort abominable du pape hérétique est figurée intégralement à droite du linteau, sans plus être abritée par le rang de palmettes car elle se passe hors de la salle conciliaire. Le pape, entouré par trois diables, est assis sur des toilettes, endroit infamant pour mourir. Il rend l'âme, figurée sous la forme d'un petit corps nu dont un diable s'empare immédiatement ${ }^{9}$.

Reprenons la Légende. Pendant ce temps, les évêques attendent le retour du pape dans la salle du concile : «Cependant, Hilaire, voyant que personne ne lui faisait place, s'arma de patience, et s'installa par terre ». Au centre du linteau, Hilaire est assis au sol, sa crosse en main. « Et aussitôt, sur décision divine, le sol sur lequel il s'était assis se souleva et l'exhaussa au niveau des autres évêques ». Ce miracle est symbolisé, sur le linteau, par la représentation d'un ange au-dessus d'Hilaire. La scène suivante montre à nouveau les trois évêques assis, avec maintenant Hilaire installé sur un fauteuil, et en face de lui, le trône vide du pape. Près d'Hilaire, un évêque se tient debout et lève sa main en direction du tympan.

\footnotetext{
${ }^{9}$ La mort du pape Léon, aux toilettes et répandant ses entrailles, s'inspire directement de celle d'Arius telle qu'elle est relatée par Athanase d'Alexandrie dans sa lettre à Sérapion (voir la traduction des Lettres à Sérapion par Joseph Lebon, Paris, Éditions du Cerf, 2007).
} 
L'histoire s'achève ainsi : "Comme on annonçait la mort misérable du pape, Hilaire se leva et confirma tous les évêques dans la foi catholique, et quand ils furent confirmés, il les renvoya chez eux ». Seule la première scène sculptée sur la gauche du linteau ne trouve pas d'explication dans la légende relatée par Jacques de Voragine. En dehors du rang de palmettes, elle se déroule par conséquent hors de la salle du concile : elle montre un homme debout, le poing levé, dans un édifice dont l'architecture témoigne d'un luxe certain.

Finalement, la composition d'ensemble du portail place sur l'axe central l'Agneau, le Christ en majesté, et en dessous dans le prolongement, la scène de l'intervention de l'ange. L'ensemble parle de lui-même : la Maiestas Domini du tympan, affirmant la nature divine du Christ, est reconnue contre les ariens dont l'hérésie est dénoncée puis corrigée au linteau.

Le récit légendaire dérive de faits historiques ${ }^{10}$. Il y eut bien un concile à Séleucie en 359 et, contrairement à ce qu'affirme Jacques de Voragine, Hilaire avait été convoqué pour y participer après qu'il eut exposé aux évêques orientaux la doctrine des Occidentaux contenue dans son ouvrage De Synodis (peut-être le livre qu'il tient à la main sur le linteau). C'est le comte Léonas qui présida ce concile au cours duquel les querelles furent incessantes entre la minorité des évêques ariens et les autres, si bien qu'un jour Léonas refusa de venir à la séance. On peut établir un parallèle entre l'absence du comte Léonas et le départ forcé du pape Léon dans la légende. Au terme du concile, la doctrine arienne fut naturellement rejetée par la majorité, mais les évêques ariens firent leur rapport à l'empereur Constance avant les autres, et ils suscitèrent dans son esprit des préventions contre la majorité du concile de Séleucie. Craignant qu'Hilaire ne cause davantage de troubles parmi les Orientaux, on le renvoya en Gaule.

\footnotetext{
${ }^{10}$ Sur le concile de Séleucie, voir notamment Paul Galtier, Saint Hilaire de Poitiers : le premier docteur de l'Église latine, Paris, Beauchesne et fils, 1960, p. 61-66.
} 


\section{Le message derrière le décor sculpté}

Dans l'iconographie, la représentation d'Hilaire au concile de Séleucie est extrêmement rare. Pour la période médiévale, un seul autre exemple est connu : il ornait le cénotaphe d'Hilaire conservé à l'abbaye Saint-Hilaire-de-la-Celle (Vienne) et daté du XII ${ }^{\mathrm{e}}$ siècle ${ }^{11}$. Dès lors, le choix de cet épisode à l'église de Semur interroge. L'architecture et le décor sculpté permettent de dater l'édifice de la seconde moitié du XII ${ }^{\mathrm{e}}$ siècle. À cette époque, l'église a déjà été donnée par les seigneurs au prieuré clunisien de Marcigny dont ils sont les fondateurs. Elle reste néanmoins le cœur de l'ensemble castral, et il est très probable que le seigneur d'alors, Damas II, ait été le commanditaire de la reconstruction. Comment expliquer l'iconographie du linteau ? Pour Gil Fishhof, ce linteau est une prise de position contre une hérésie, mais pas nécessairement l'hérésie arienne : en effet, il était courant, aux $\mathrm{XI}^{\mathrm{e}}$ et $\mathrm{XII}^{\mathrm{e}}$ siècles, de qualifier une hérésie d'arienne même si, en réalité, sa doctrine était tout autre. L'histoire d'Hilaire dut servir de couverture, d'exemplum, à une autre histoire édifiante.

De fait, ici, l'exploit d'Hilaire à Séleucie vise à glorifier Hugues de Semur, l'arrière-grand-oncle du commanditaire de Saint-Hilaire, Damas II, lequel conservait naturellement le souvenir de son prestigieux ancêtre. Mais la question rebondit : quel lien établir entre l'abbé de Cluny et saint Hilaire ? Hugues était étroitement associé aux papes Grégoire VII puis Urbain II (tous deux de formation clunisienne) dans la conduite de la réforme grégorienne. En particulier, tout en assurant un rôle de médiateur, il avait pris parti pour le pape dans la querelle des investitures qui opposait Grégoire VII à l'empereur Henri IV. Ce dernier fit introniser l'antipape Clément III en 1084 et tous deux furent considérés par la papauté comme hérétiques. Le lien avec le linteau de l'église de Semur se dessine : le pape Léon y incarne l'antipape Clément et, dans la foulée, le

\footnotetext{
${ }^{11}$ Les faces latérales du cénotaphe, qui portaient la représentation de la mort du pape Léon et de l'intervention de l'ange, sont connues uniquement par des dessins de Roger de Gaignières (Paris, BNF, Cabinet des Estampes, latin 17042, f ${ }^{\circ}$ 9-10).
} 
mystérieux personnage au poing serré représenté dans un riche palais représente l'empereur arien Constance, si l'on se place au temps d'Hilaire, et naturellement l'empereur Henri IV, si l'on se situe au temps de la réforme grégorienne. Par son action exemplaire, Hugues de Semur est un nouvel Hilaire combattant les hérétiques de son temps. Ainsi, en associant Hugues à Hilaire, le seigneur de Semur ranimait la mémoire de ce membre éminent de la famille, décédé en 1109 , pour célébrer la grandeur de la lignée et, par là, asseoir ou afficher son autorité et sa réputation. D'ailleurs, il s'inscrivait par cet acte dans la continuité de celui de ses prédécesseurs, peut-être Geoffroy IV, qui avait choisi de placer la chapelle sous le vocable d'Hilaire, une titulature rare dans les diocèses de Bourgogne méridionale ${ }^{12}$.

La lecture du linteau par Gil Fishhof révèle la profondeur d'esprit qui présida à sa conception, en dépassant les critiques qui lui étaient portées jusqu'alors : l'iconographie du portail occidental était un moyen subtil de mettre en valeur la renommée du lignage. Le linteau achevé glorifiait et la Trinité et le noble seigneur du lieu. On concevra cependant qu'il s'adressait sans doute aux esprits les plus fins, formés par exemple aux écrits de Placide de Nonantola qui avait fait en 1111 le lien entre Hilaire d'une part, et les papes Grégoire VII et Urbain II d'autre part ${ }^{13}$. À l'inverse, l'architecture de l'église, elle, délivre le même message mais par des procédés visuels accessibles au plus grand nombre. L'église Saint-Hilaire permet de saisir, jusque dans les repentirs du chantier, la pensée du commanditaire.

\footnotetext{
${ }^{12}$ Voir note 2.

${ }^{13}$ Placide de Nonantola, Liber de honore Ecclesiae, éd. Ernst Dümmler et al., dans Libelli de lite Imperatorum et Pontificum saeculis XI et XII conscripti, Hannover, Hahn (Monumenta Germaniae Historica), vol. 2, 1892, p. 637-638.
} 


\section{Le chantier, témoin discret des mérites de la famille}

Partons de l'église telle qu'elle se présente. Longue de $37 \mathrm{~m}$ et large de $16 \mathrm{~m}$, l'église présente une nef à trois vaisseaux de quatre travées ouvrant sur un transept très peu saillant (fig. 3). L'ampleur des dimensions et la présence d'un transept distinguent Saint-Hilaire des autres églises paroissiales romanes du Brionnais, généralement plus modestes. Le chœur est développé lui aussi, et deux bas-côtés encadrent la travée de chœur qui ouvre sur une abside encadrée d'absidioles. Le vaisseau central de la nef présente lui aussi des dispositions uniques en Brionnais puisque son élévation compte trois niveaux : grandesarcades, arcature et fenêtres hautes.

En 1920, dans un article du Bulletin monumental, André Rhein fut le premier à relever une irrégularité surprenante : un double cordon de perles court dans le transept, entre les deux niveaux de l'élévation en passant par les tailloirs des piles de la croisée, et se poursuit dans le vaisseau central de la nef sur une vingtaine de centimètres avant de s'interrompre brutalement ${ }^{14}$ (fig. 4). Il reprend environ $40 \mathrm{~cm}$ plus haut, au-dessus des grandes-arcades. André Rhein interprétait ce décalage comme la preuve d'un changement de projet. Il supposait qu'on prévoyait de construire une nef plus basse et dont la voûte aurait reposé sur le cordon inférieur. Cette hypothèse le séduisait car elle permettait à la baie ouest du tambour de la coupole, qui ouvre actuellement dans le vaisseau central, de prendre jour sur l'extérieur.

En 1956, Raymond Oursel reprend mot pour mot l'analyse d'André Rhein et tente de l'interpréter à la lumière de l'histoire de la seigneurie ${ }^{15}$. En revanche, il ne propose pas d'étude architecturale qui étaierait la proposition d'une nef plus basse. Un élément décisif montre pourtant que l'intuition d'André Rhein était fondée. Chacun des bras du transept est percé, à l'ouest, par une baie donnant dans

\footnotetext{
${ }^{14}$ André Rhein, «Église de Semur-en-Brionnais », art. cit., p. 188.

${ }^{15}$ Raymond Oursel, Les Églises romanes de l'Autunois et du Brionnais, op. cit., p. 291.
} 
les combles des bas-côtés. La baie du bras nord permet l'accès aux combles et au clocher, tandis que celle du sud est aveugle. L'observation de cette baie sud depuis les combles montre que son encadrement est parfaitement appareillé. Elle fut donc conçue pour être ouverte, avant d'être rendue aveugle par un bouchage. Ainsi, lorsqu'on édifiait les bras du transept, on prévoyait d'élever une nef plus basse permettant aux baies du mur ouest d'ouvrir sur l'extérieur.

Quelle nef prévoyait-on de construire à l'origine ? Il devait s'agir d'une nef à trois vaisseaux, aussi large que la nef actuelle, car une nef unique s'accorderait mal avec des parties orientales très développées. L'amorce du cordon de perles et les baies ouest du transept nous guident pour restituer l'élévation du vaisseau central et ses proportions. On peut raisonnablement supposer qu'on prévoyait de reprendre dans le vaisseau central l'élévation des bras du transept. Il faut donc restituer une élévation bipartite, avec des grandes-arcades au premier niveau et des fenêtres hautes au second (fig. 5) ${ }^{16}$. Le double cordon de perles aurait marqué la séparation des deux niveaux. Un berceau, peut-être brisé comme dans les croisillons, devait couvrir le vaisseau. Pour respecter la hauteur imposée par les baies du mur ouest du transept, les fenêtres hautes de la nef devaient nécessairement être en pénétration dans le berceau ${ }^{17}$. Cette nef, telle que nous la restituerions volontiers, s'accorderait mieux avec les volumes des parties orientales que la nef actuelle, l'alignement des toits serait plus harmonieux et le clocher émergerait davantage; l'église serait finalement plus élancée, moins trapue (fig. 6).

Lors du changement de projet, les bras, la croisée et le tambour de la coupole du transept devaient être construits, en revanche la nef était à peine amorcée, si bien que la nef actuelle appartient dans son intégralité au nouveau

\footnotetext{
${ }^{16}$ L'hypothèse émise par André Rhein d'une nef ne comprenant qu'un niveau de grandesarcades, comme à Iguerande, est moins satisfaisante.

${ }^{17}$ En Brionnais, l'église de Châteauneuf offre un point de comparaison intéressant. En effet, ses parties orientales ressemblent beaucoup à celles de Saint-Hilaire ; or les fenêtres hautes du vaisseau central de la nef sont construites en pénétration dans le berceau.
} 
projet. Si le cordon de perles a été légèrement remonté, c'est pour donner un peu plus d'ampleur aux grandes-arcades, car dans les élévations tripartites, des proportions précises sont à respecter entre les différents niveaux pour obtenir l'élan vertical souhaité. Si l'on avait maintenu les grandes-arcades à la hauteur initialement prévue, l'élévation aurait paru tassée, et nous aurions perdu cet infime indice d'un projet abandonné.

La nouvelle nef répond à un double objectif : monumentaliser l'édifice, et ce en lui donnant une apparence clunisienne. En effet, l'élévation tripartite, le tracé brisé des arcs et du berceau, les pilastres cannelés, l'absidiole en encorbellement au revers de la façade attestent que le modèle suivi a été la troisième abbatiale de Cluny commencée en 1088. Le seul écart que les constructeurs se sont accordé par rapport à l'originale fut de laisser l'arcature du second niveau ouverte sur les combles, alors qu'à Cluny elle était aveugle. Aujourd'hui, cette différence est moins perceptible car, à Saint-Hilaire, l'arcature a été bouchée par une maçonnerie de briques. C'est par ces mêmes combles qu'on rejoint l'absidiole en encorbellement située au revers de la façade et dont la fonction demeure une énigme (fig. 7). Cet élément est caractéristique des églises clunisiennes où l'absidiole de la chapelle haute de l'avant-nef fait saillie dans la nef. Ce motif architectural, probablement conçu dans l'abbatiale Cluny II, est repris à Cluny III et est actuellement visible dans les prieurales de Payerne et de Romainmôtier. À Semur, il est difficile d'accéder à cet espace, tant les combles sont bas et sombres. La charpente semble être dans sa position d'origine, car des éléments de la maçonnerie ont été spécialement conçus pour porter la panne faîtière. On imagine donc mal un prêtre se rendre dans l'absidiole. Et d'ailleurs, pourquoi s'y rendrait-il ? Dans les églises monastiques, cet espace est associé à la célébration des messes pour les défunts, mais Saint-Hilaire n'est pas une église monastique. L'absidiole en encorbellement ne remplirait donc pas de fonction liturgique, mais symboliserait visuellement et de manière forte le lien unissant les 
seigneurs de Semur à l'abbaye clunisienne ${ }^{18}$. Ce lien ne se résume d'ailleurs pas au personnage d'Hugues car, après que ce dernier eut fondé le prieuré de Marcigny avec son frère Geoffroy sur des terres proches du château, plusieurs hommes de la famille en devinrent prieurs tandis que les femmes y entraient comme moniales.

L'architecture distingue complètement Saint-Hilaire de toutes les autres églises du Brionnais. Comme pour le linteau, le parti adopté pour l'église est une manière pour le seigneur de rappeler que l'illustre abbé Hugues de Semur figurait dans son lignage. Le parti architectural, l'iconographie du linteau couronnant le choix du vocable, Saint-Hilaire, se révèlent donc politiques : affirmer le prestige et la puissance de la famille seigneuriale en rappelant un glorieux héritage. L'exemple de Semur illustre un moyen utilisé par les grands pour afficher leur respectabilité et ainsi accroître une notoriété essentielle à leur autorité.

18 Jean-Noël Barnoud, Nicolas Reveyron, Gilles Rollier, Paray-le-Monial, Paris, Zodiaque, 2004, p. 204. 


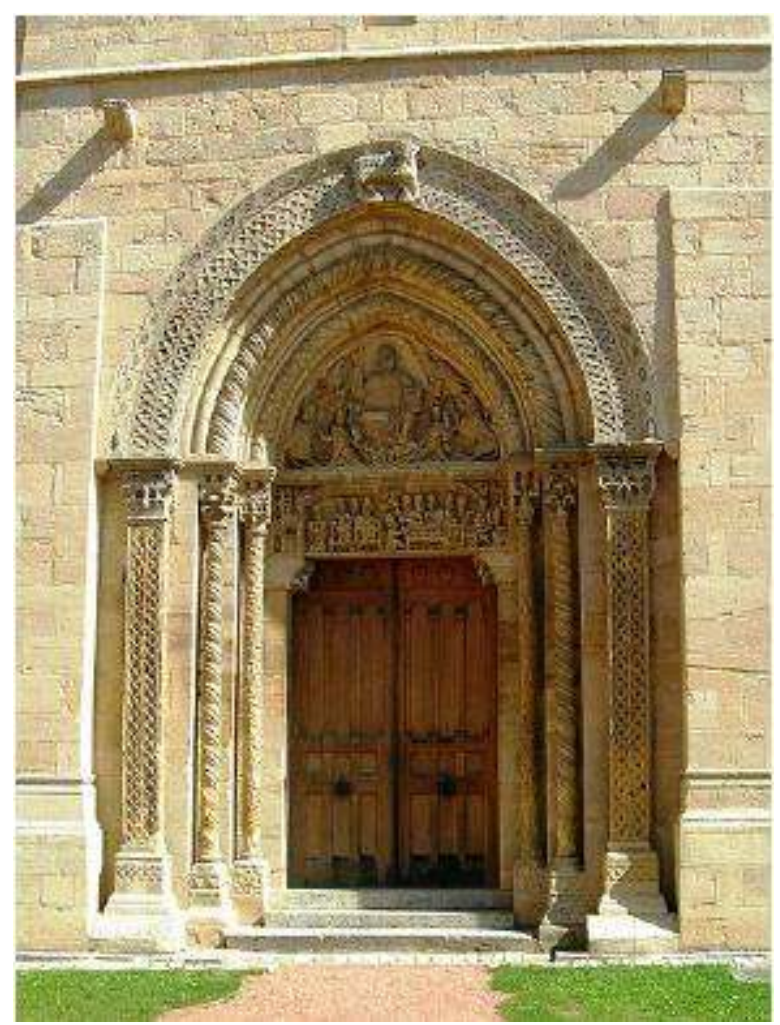

Fig. 1 : Semur-en-Brionnais, église Saint-Hilaire, portail ouest (C) Wikimedia Commons / Jan Sokol.

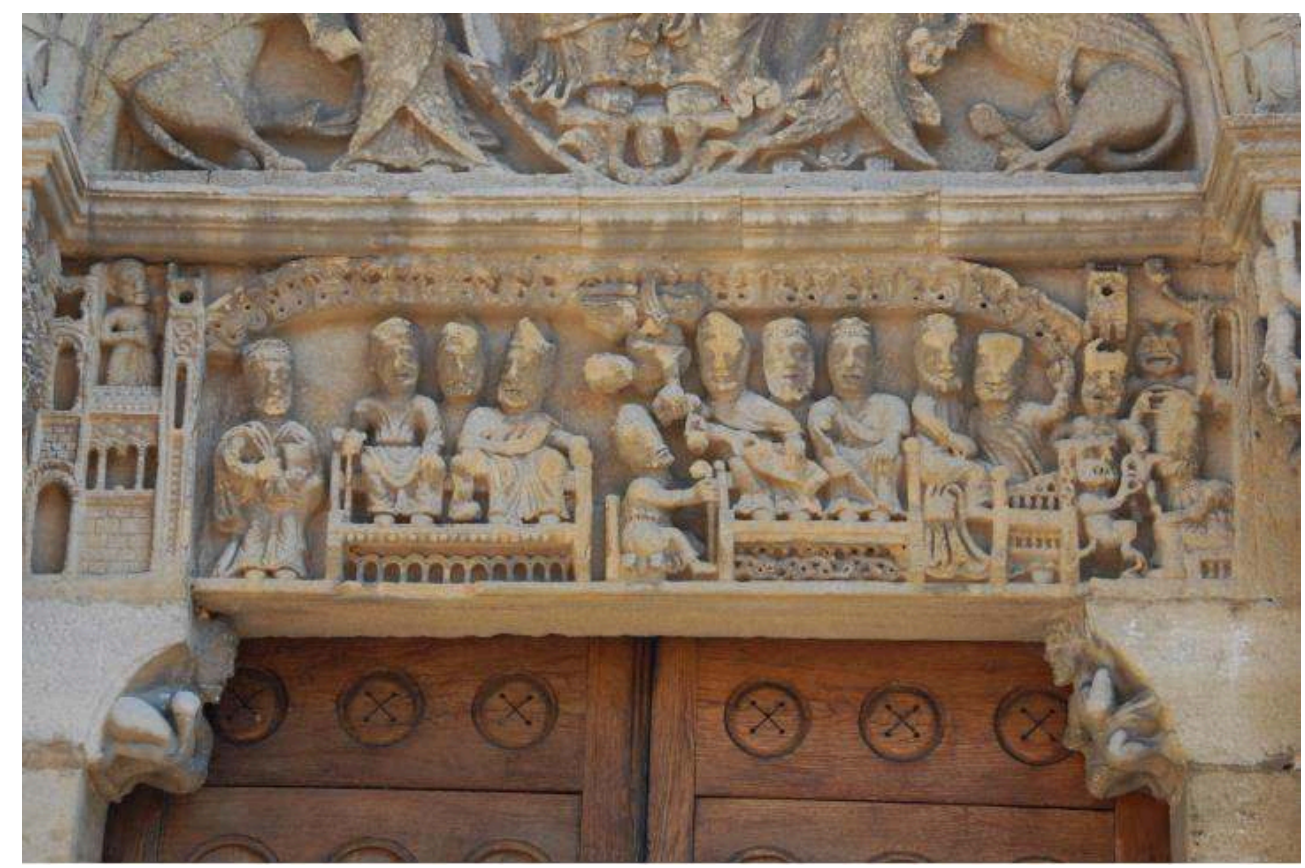

Fig. 2 : Semur-en-Brionnais, église Saint-Hilaire, linteau du portail ouest, (C) Wikimedia commons / Jochen Jahnke. 


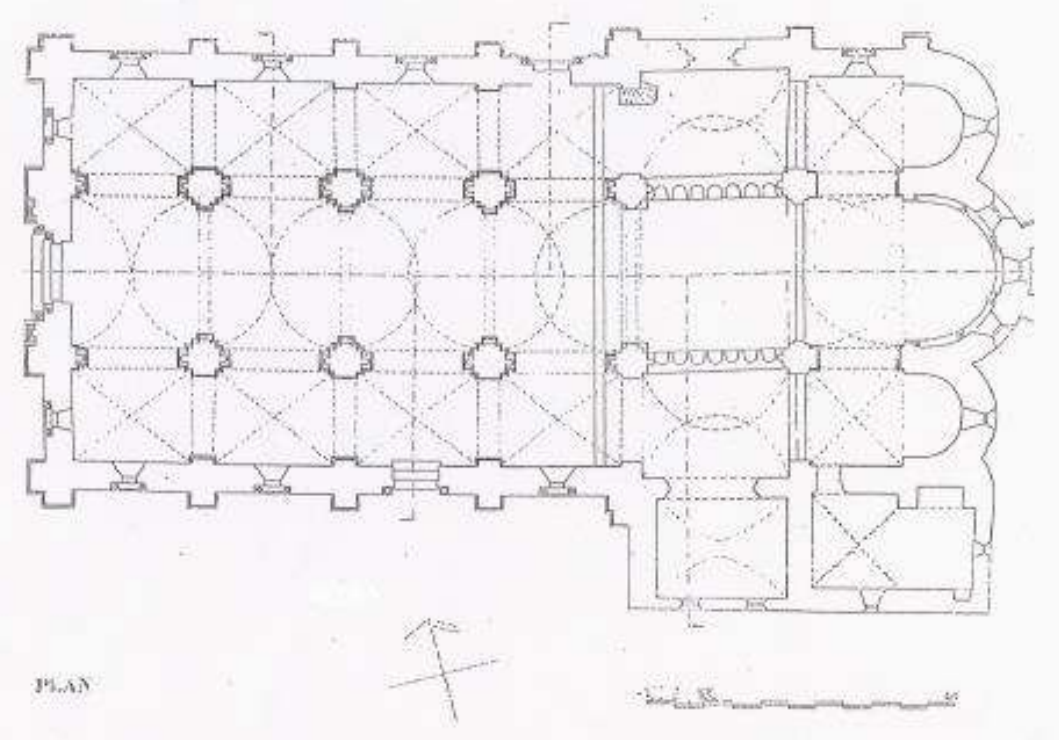

Fig. 3 : Semur-en-Brionnais, église Saint-Hilaire, plan @ C CEP.

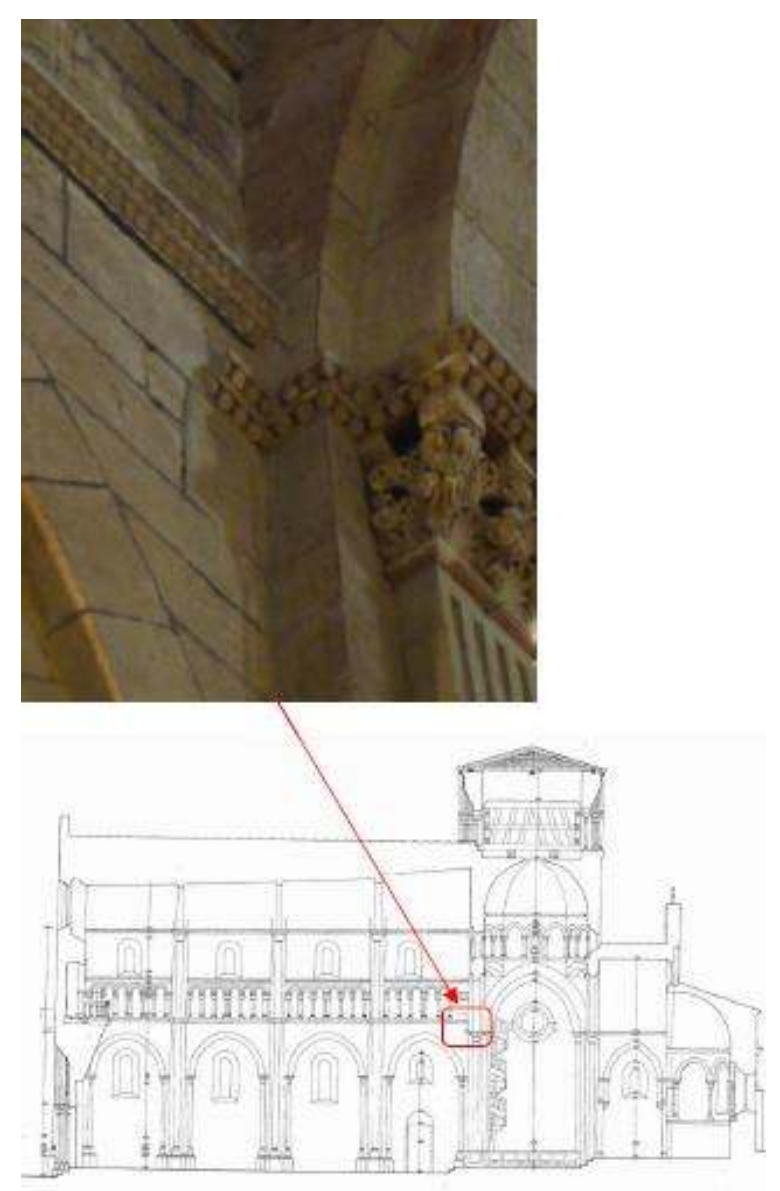

Fig. 4 : Semur-en-Brionnais, église Saint-Hilaire.

Le décalage entre les cordons de perles à la jonction entre le transept et la nef. Photographie de l'auteur / Coupe longitudinale (C) CEP. 


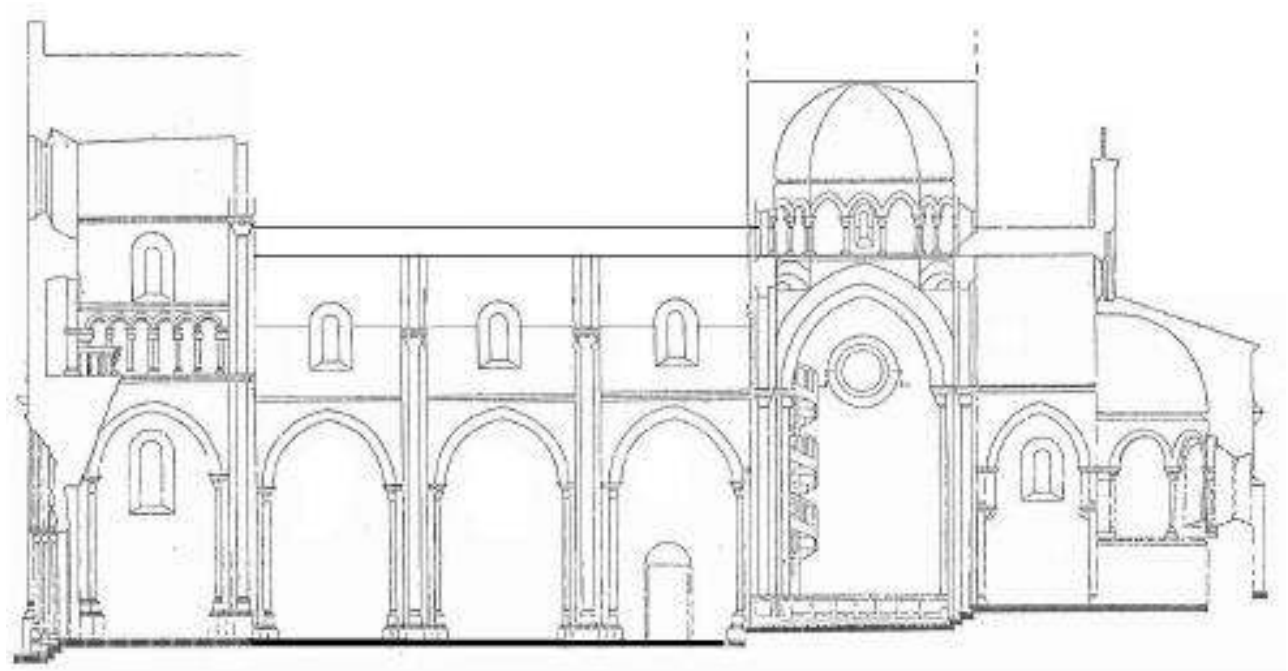

Fig. 5 : Semur-en-Brionnais, église Saint-Hilaire. Coupe longitudinale. La première travée de la nef correspond à l'église actuelle.

Les trois autres travées sont une proposition de restitution du projet abandonné. (C) CEP. Restitution du premier projet par A. Nicolier)

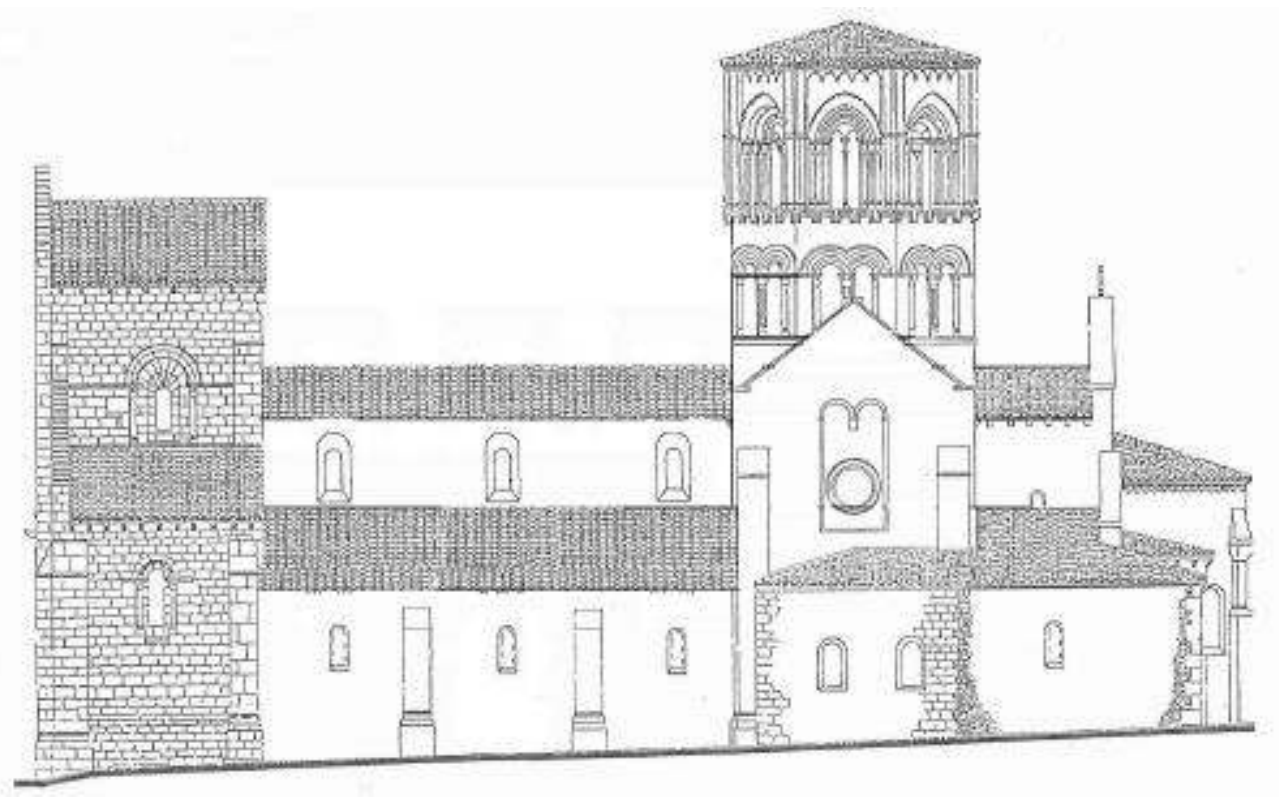

Fig. 6 : Semur-en-Brionnais, église Saint-Hilaire. Vue en élévation du côté sud. La première travée de la nef correspond à l'église actuelle.

Les trois autres travées sont une proposition de restitution du projet abandonné. (C) CEP. Restitution du premier projet par A. Nicolier) 


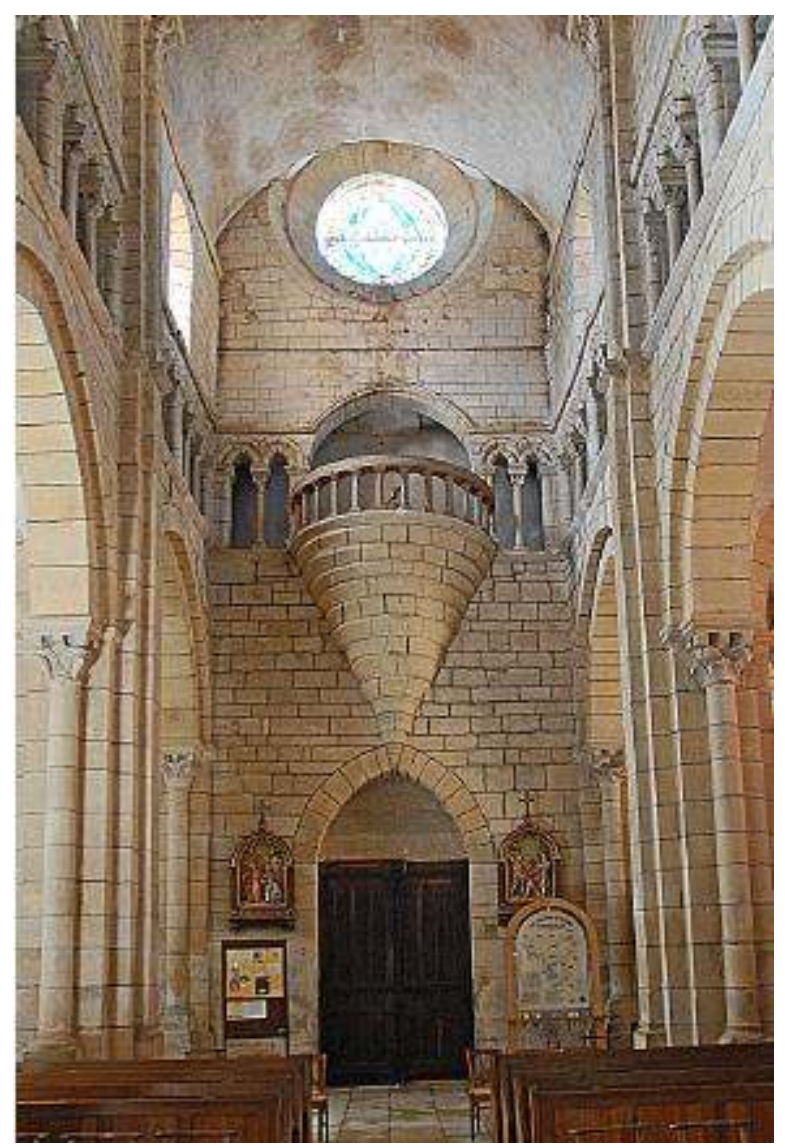

Fig. 7 : Semur-en-Brionnais, église Saint-Hilaire, nef, revers de la façade ouest. (C) Wikimedia commons / Jochen Jahnke. 
